\title{
Spatial Dependency in Employment Growth in NC Counties
}

\author{
Ella Scott
}

\section{A. Introduction}

Economic impact analysis has a significant role in policy making for the governments. It can increase/decrease the economic opportunities for the residents of each area. Many researchers have been studying the effect of employment growth in different regions on increasing/decreasing of employment opportunities in other regions in close proximity.

In this project, I will study whether employment growth in one county can affect activities in adjacent counties within the state of North Carolina. In other words, I want to explore whether growing counties are close to each other or not by using spatial autocorrelation techniques.

The unit of analysis of this research is counties in NC. I use percentage change in employment of each county between 2010 and 2015 data, collected from BEA website to test my hypothesis.

\section{B. Purpose of the study}

- To find whether there is any spatial autocorrelation between different counties in NC in terms of economic activity and provide this information to decision makers for their regional analyses

- Economic Impact Assessment to assess how activities in adjacent counties within the state impact conditions and processes inside of North Carolina

- Whether growing counties are close to each other

- Estimates of county-based, project-specific employment spillovers to/from one county 
The result of this research will be of an interest of policy makers, and in general, decision makers in the state of North Carolina to consider the effects of increasing employment in different regions and how to use it for their further decisions.

\section{Literature Review}

\section{Theory: Definition of Spatial Autocorrelation}

Wherever location matters and influences the behavior of another entity, spatial autocorrelation is likely to be present (Dubin, 1998). Autocorrelation means that a variable is related to itself. Based on the first law of geography by Waldo Tobler "Everything is related to everything else, but near things are more related than distant things." (Tobler 1970), so autocorrelation states that those things that are closer to each other are more likely to have similar values than those things that are far from each other. The spatial structure of the data might be in a way that they have spatial autocorrelation, and because of that, it is possible to predict the value of a location based on the value of a nearby location.

Although related to correlation, spatial autocorrelation has its own concept. While correlation defines relationships among variables, "spatial autocorrelation shows the correlation within variables across georeferenced space" (Getis, 2008). Main assumption of Ordinary Least Squares is that the error terms are independent under the null hypothesis (Cliff \& Ord, 1972) however, this statistic is used to measure spatial autocorrelation in the georeferenced data. In these situations, the error term needs to be spatially independent but it is not.

The concept of spatial autocorrelation began to flourish in the late 1950s at the University of Washington by Michael F. Dacey, William L. Garrison, and Edward Ullman, however the term, 
spatial autocorrelation, was first used by Andrew D. Cliff and Keith Ord in 1968 in their book “The Problem of Spatial Autocorrelation” (Getis, 2008)

\section{Measure of Spatial Autocorrelation}

Several measures have been introduced to quantify spatial autocorrelation. Global Moran's I (Moran 1950), Geary's C (Geary 1954), Black-White Join Counts and Getis-Ord G Statistic are three tools that measure spatial autocorrelation that will be discussed in this section.

\subsection{Black-White Join Counts}

Black-White join Counts is a measure of spatial autocorrelation. When the variable of interest is binary, a join count analysis can be used to assess the degree of clustering or dispersion. It can be mapped into two colors of black and white. It also should be applied to polygons only. It requires a contiguity matrix for the polygons and it measures the number of borders ("joins") of each type relative to total number of borders.

If the number of BW joins is significantly lower than what we would expect by chance, join count statistics display positive spatial autocorrelation (clustering). If the number of BW joins is significantly higher than what we would expect by chance there is negative spatial autocorrelation (dispersion). And finally, if the number of BW joins is approximately the same as what we would expect by chance there is no spatial autocorrelation (random pattern).

\subsection{Global Moran's I}

Global Moran's I measures spatial autocorrelation based on feature locations and feature values. It analyzes if the pattern of that feature is clustered, dispersed, or random. It basically measures similarity among features based on their location and their attributes and defines global spatial autocorrelation (Liu et al. 2015). 
Global Moran's I is an inferential statistic that the null hypothesis states that the attributes are randomly distributed among the features in the location of the study. It can be computed as the following equation:

$$
I=\frac{n}{\sum_{i} \sum_{j} w_{i j}} \frac{\sum_{i} \sum_{j} w_{i j} z_{i} z_{j}}{\sum_{i} z_{i}^{2}},
$$

where $z_{i}$ and $z_{j}$ are derivations of observations from the mean evaluated at sites $i$ and $j$, $n$ is the number of observation sites;

$w_{i j}$ is the defined weight based on the spatial proximity between sites $i$ and $j$ (Liu et al. 2015).

I statistics varies between -1 and +1 , where values between 0 and -1 shows a negative association between variables, when $\mathrm{N} \rightarrow \infty$ then it approaches to 0 that indicates there is no correlation between variables, and values between 0 and +1 shows a positive association between variables.

This equation can be used to measure spatial autocorrelation for scalar variables. The similarity of observations is evaluated by comparing each observation to the overall mean. In a positive spatial autocorrelation pattern, observations close in space will tend to be higher/lower than the mean, leading to a positive I (Anselin, 2010). 


\subsection{Geary's C}

Geary's C also can be used to measure spatial autocorrelation, and dislike the Global Moran's I, it is a measure of dissimilarity among the features in the study area. The larger the amount of Geary's $\mathrm{C}$, the less similar the features are. It can be computed as the following equation:

$$
\mathcal{C}=\frac{(n-1) \sum_{i} \sum_{j} w_{i j}\left(y_{i}-y_{j}\right)^{2}}{2\left(\sum_{i} \sum_{j} w_{i j}\right) \sum_{i}\left(y_{i}-\bar{y}\right)^{2}}
$$

where $y_{i}$ is the value of a variable for the $i$ th observation, $y$ hat is the sample mean and $w_{i j}$ is the spatial weight of the connection between $i$ and $j$.

Its range is from 0 to infinity. Between 0 and 1there is positive spatial autocorrelation, equal to 1 there is no spatial autocorrelation, and larger than 1 there is negative spatial autocorrelation (dispersion) (Jong et al., 1984).

\subsection{Getis-Ord G Statistic}

The Getis-Ord Gi* statistic (Gi*) (Getis and Ord, 1992, 1996; Mitchell, 2005; Ord and Getis, 1995) is a method for analyzing whether the attributes of spatial data have any location-related tendency (clustering). It is also known as hot-spot analysis.

This method is adapted from the General G-statistic (Getis and Ord, 1992), and it quantifies the degree of global spatial autocorrelation over an area. It can be computed as the following equation: 


$$
G=\frac{\sum_{i=1}^{n} \sum_{j=1}^{n} w_{i, j} x_{i} x_{j}}{\sum_{i=1}^{n} \sum_{j=1}^{n} x_{i} x_{j}}, \forall j \neq i
$$

Where $x_{i}$ and $x_{j}$ are attribute values for features $i$ and $j$, and $w_{i j}$ is the spatial weight between feature $i$ and $j . n$ is the number of features in the dataset and indicates $\forall j \neq i$ that features $i$ and $j$ cannot be the same feature.

\section{Applications}

Spatial autocorrelation applies to many areas of study such as transportation mode choice, land cover change, terrain analysis and climate studies, and also discrete objects such as areal units like census tracts or counties. In this example, spatial closeness is measured based on proximity of polygons (Liu et al. (2015).

Some scholar analyze the spatial autocorrelation among multi-location events and they call it the examination of the correspondence of spatial clusters. By studying the patterns of paired zerodimensional point locations, they developed cluster correspondence analysis to assess the spatial clustering patterns of one-dimensional vectors.

\section{Software}

In the late 1980s and early 1990s, there were not specific spatial data analytical software that made the application of it limited in empirical work (Anselin and Griffith 1988; Haining 1978; Goodchild et al. 1992). However, in the beginning of the $21^{\text {st }}$ century this situation has been changed. Currently there are several software to measure spatial autocorrelation, that include:

- ArcGIS 
- GeoDa (https://geodacenter.asu.edu/)

- $\mathrm{R}$ (spdep package)

\section{Summary}

In summary, spatial autocorrelation shows the correlation within variables across georeferenced space. Among all the measures of spatial autocorrelation, Moran's I has been used a lot more in empirical work than Geary's C, Black-White Join Counts, and Getis-Ord G Statistic. ArcGIS, R, and GeoDa are popular software applications to do spatial autocorrelation.

\section{Economic Impact Assessment}

As I pointed out earlier in this paper, I am going to explore the employment growth in each region (counties of North Carolina) and its spillovers effect on other adjacent regions. In other words, I will look at the Economic Impact Assessment Model to include the capability to assess how activities in adjacent counties within the state of North Carolina impact conditions and processes inside of North Carolina (i.e., estimates of county-based, project-specific employment spillovers to/from one county).

\section{E. Technical Approach}

To see if there is any spatial autocorrelation and the spatial dependency among the counties I will start with spatial autocorrelation analyses and then I will estimate an OLS regression model, and by getting the residuals of the model I will understand if there is spatial dependency and get to the spatial models such as spatial lag model or spatial error model.

To estimate employment spillovers between counties within the state of North Carolina, I use spatial econometric techniques to estimate the following base model:

$\Delta Y_{i, t+5}=\beta 0+\rho W \Delta Y_{i, t+5}+\beta 1 H C_{i, t}+\beta 2 M A N F_{i, t}+\beta 3 I N C_{i, t}+\beta 4 M E T R O_{i, t}+\varepsilon_{i, t}$ 
Where $\Delta Y$ is the difference in final $(t+5)$ and initial year employment $(t)$ county $i$ at time $t+5$. This specification controls for neighboring county employment by including the spatial lag of the difference in employment over the same five year period. $W$ is the first-order queen contiguity spatial weights matrix and $\rho$ is the spatial autoregressive coefficient. The spatial lag model is employed as firms engaged in similar industries tend to locate near one another. Furthermore, due to commuting relationships between counties it is expected that local economies may see increased demand for (non-basic) goods and services from residents gaining employment in neighboring counties.

I also control for the employment shares of manufacturing $(M A N F)$ to control for product demand across cities. As suggested by existing literature I include a measure of per capita income (INC) to control for effects of differences in factor prices. I also include a dummy to indicate whether a county is urban or rural (METRO). The cross sections time that I use in this study 2010-2015.

From this estimation procedure, (first-order) county-specific multipliers are extracted to quantify how each neighboring country is affected in terms of expected employment gains/losses when a county is experiencing an employment increase/decrease (e.g., if Mecklenburg County adds 1,000 how many jobs are Cabarrus County likely to gain due to spatial linkages between the two counties).

\section{F. Data}

I have collected data for 100 counties of NC employment for 2010 and 2015 from various sources that are listed following:

- EMP2010 and EMP2015: Total Private Non-Farm employment (BEA).

- MANF2010: share of manufacturing employment (NAICS 31-33), Employment by 2-digit NAICS industry (BEA). 
- INC2010: Per capita income (Census/ACS).

- METRO: if the county is in a metro area or in a nonmetro area.

- Map of counties of North Carolina: (Census/Tiger) (shape file)

I have also calculated the following variables:

- Spatial Weight Matrix from GeoDa:

- First-order queen contiguity spatial weights matrix

- Percentage change in employment between 2010 and 2015:

- $(($ EMP2015-EMP2010)/EMP2010)*100

- Residuals of two OLS regressions

\section{G. Results}


Based on map 1 that is the analysis of employment in 2010, and also the following spatial autocorrelation test of Moran's I, we can see there is spatial autocorrelation among employment in counties of NC.

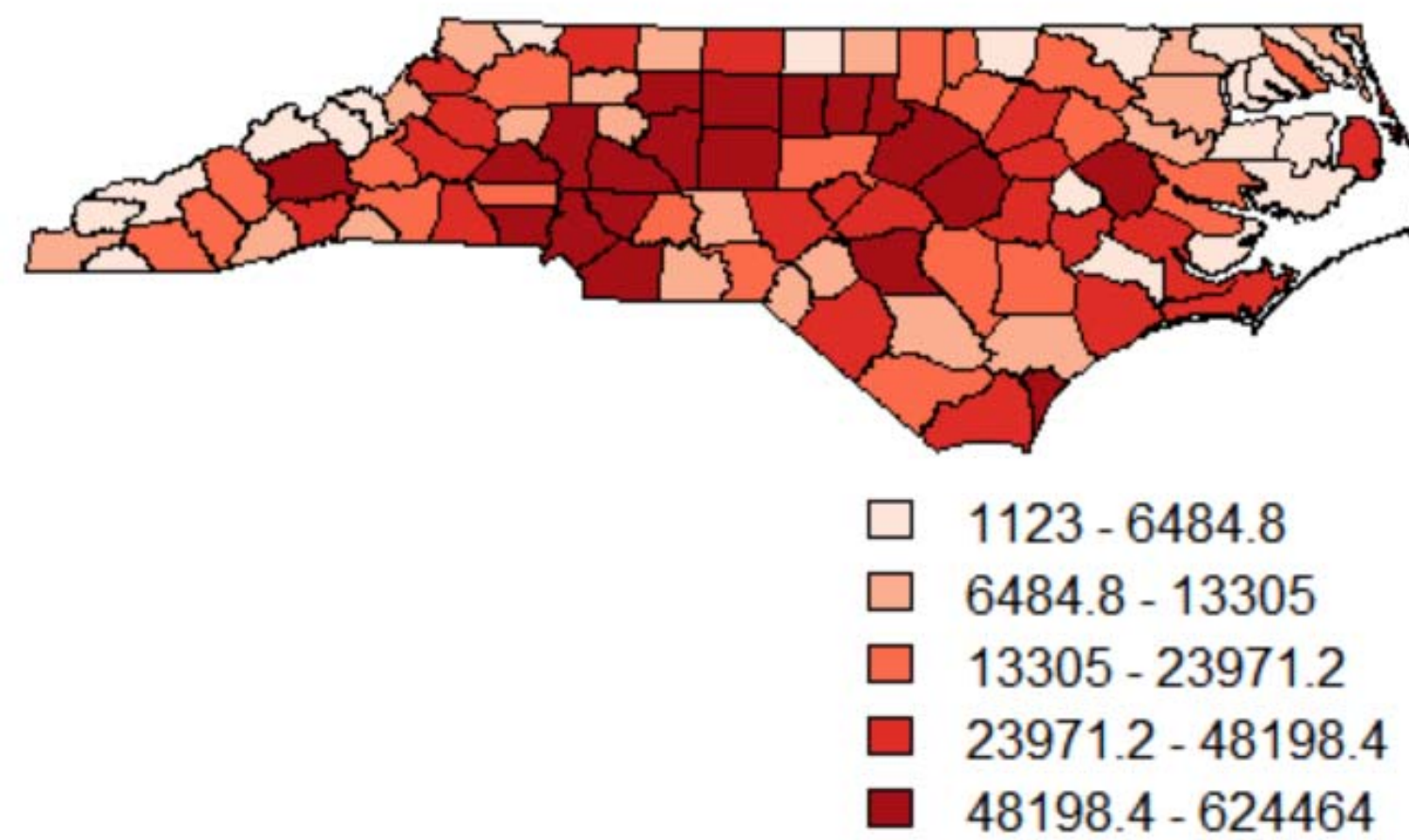

Map 1: Employment in 2010

Global Moran's I is for global spatial autocorrelation and it measures similarity. The result of global Moran's I in 2010 shows that the p-value is less than 5\% Confidence Interval, so we reject the null hypothesis that employment is randomly distributed, and we accept that there is spatial autocorrelation in our study area. Also, based on the result, since the Moran I statistic is greater than the expectation value, we have positive autocorrelation and there are clustering of employment in counties of NC. 


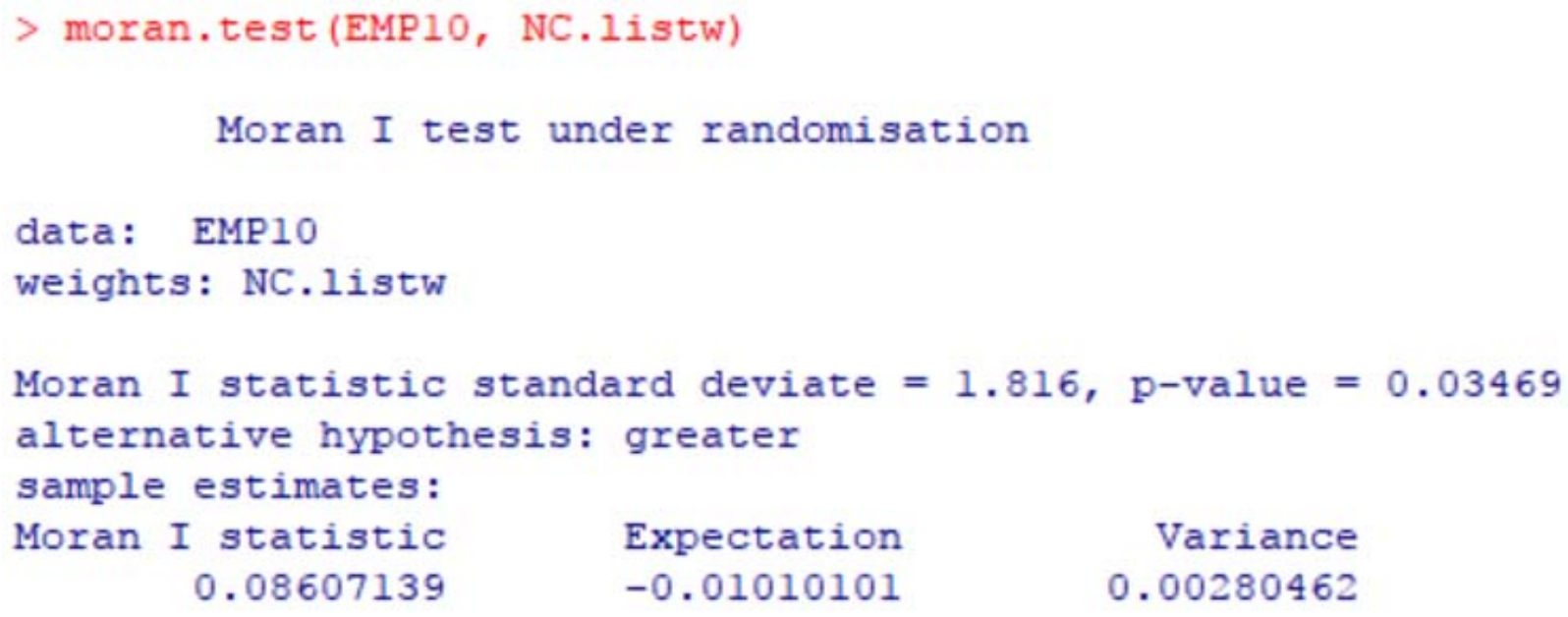

Based on map 2 that is the analysis of employment in 2015, and also the following spatial autocorrelation test of Moran's I, we can see there is spatial autocorrelation among employment in counties of NC in 2015 too. And same as 2010, the result of global Moran's I in 2015 shows that the p-value is less than $5 \%$ Confidence Interval, so we reject the null hypothesis that employment is randomly distributed, and we accept that there is spatial autocorrelation in our study area. Also, based on the result, since the Moran I statistic is greater than the expectation value, we have positive autocorrelation and there are clustering of employment in counties of NC in 2015. 

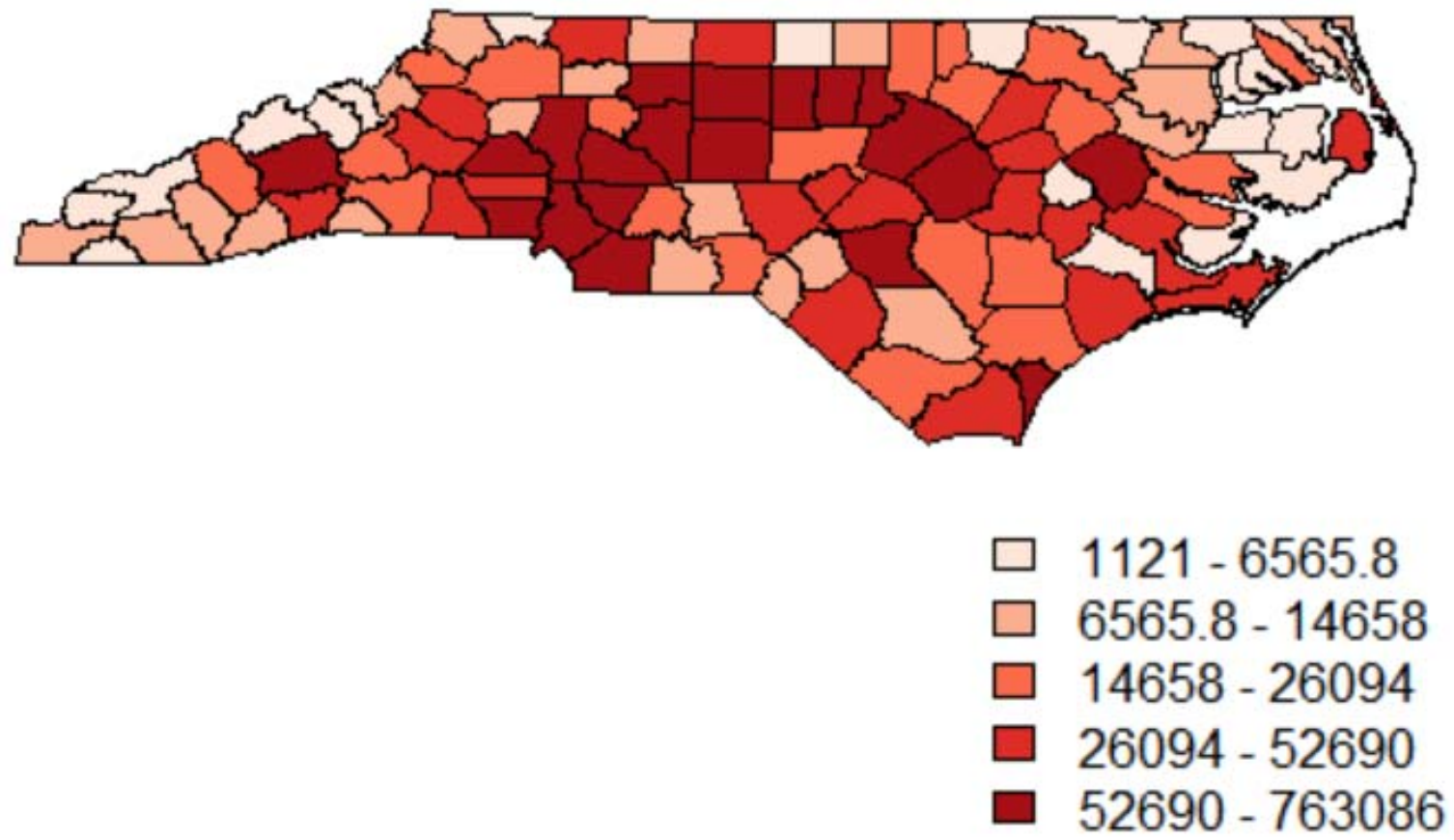

Map 1: Employment in 2015

$>$ moran.test (EMP15, NC.listw)

Moran I test under randomisation

data: EMP15

weights: NC.listw

Moran I statistic standard deviate $=1.7646$, p-value $=0.03882$ alternative hypothesis: greater

sample estimates:

Moran I statistic

Expectation

Variance

0.081596668

$-0.010101010$

0.002700463 
Here we are looking at percentage change of employment between 2010 and 2015 calculated from the employment of 2010 and 2015. Based on map 3 that is the analysis percentage change of employment between 2010 and 2015, and the following spatial autocorrelation test of Moran's I, we can see there is spatial autocorrelation percentage change of employment between 2010 and 2015 in counties of NC. And same as 2010 and 2015, the result of global Moran's I shows that the p-value is less than $5 \%$ Confidence Interval, so we reject the null hypothesis that percentage change of employment between 2010 and 2015 is randomly distributed, and we accept that there is spatial autocorrelation in our study area. Also, based on the result, since the Moran I statistic is greater than the expectation value, we have positive autocorrelation and there are clustering of percentage change of employment in counties of NC.

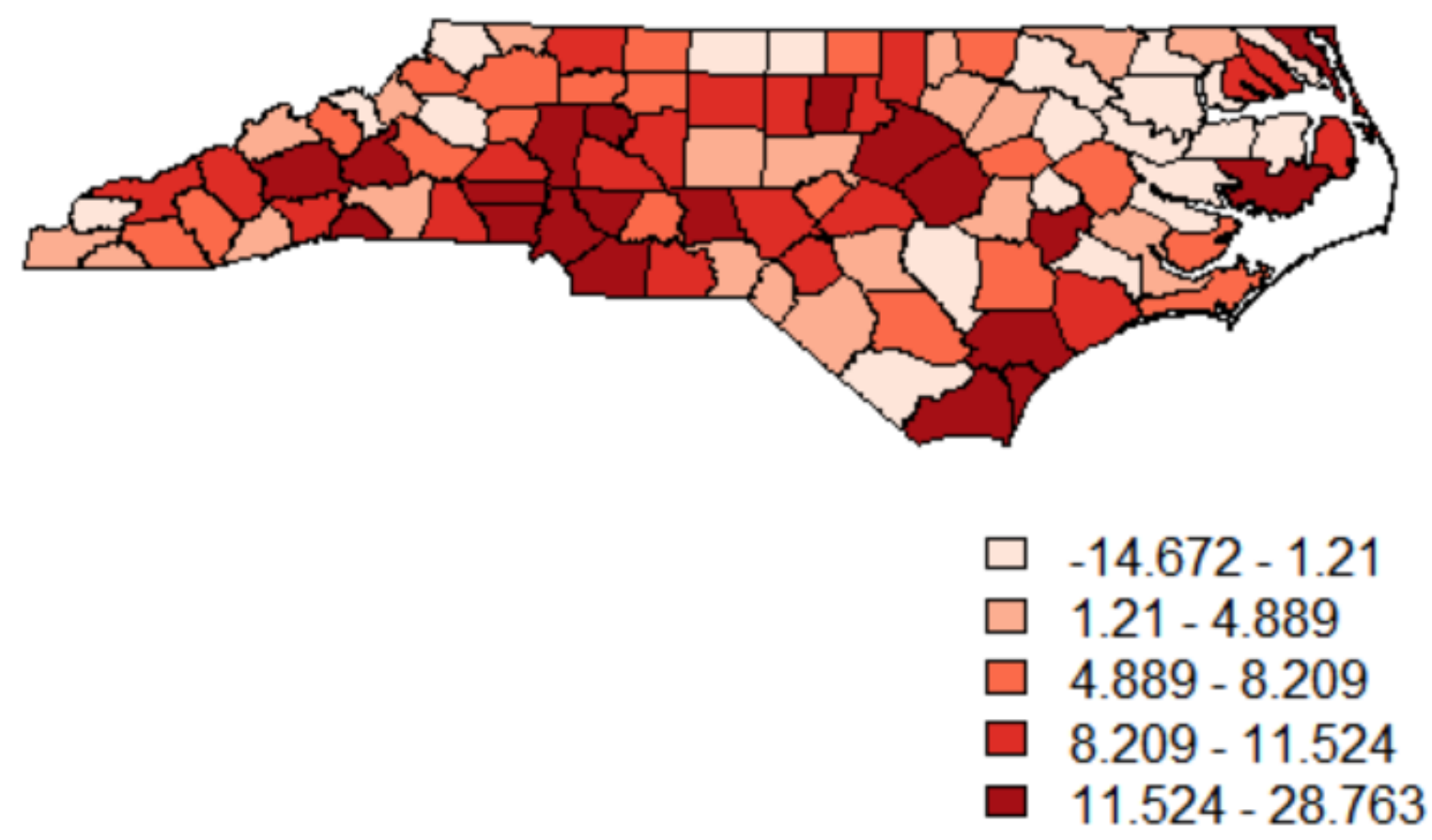

Map 1: Percent Change in Employment between 2010 and 2015 


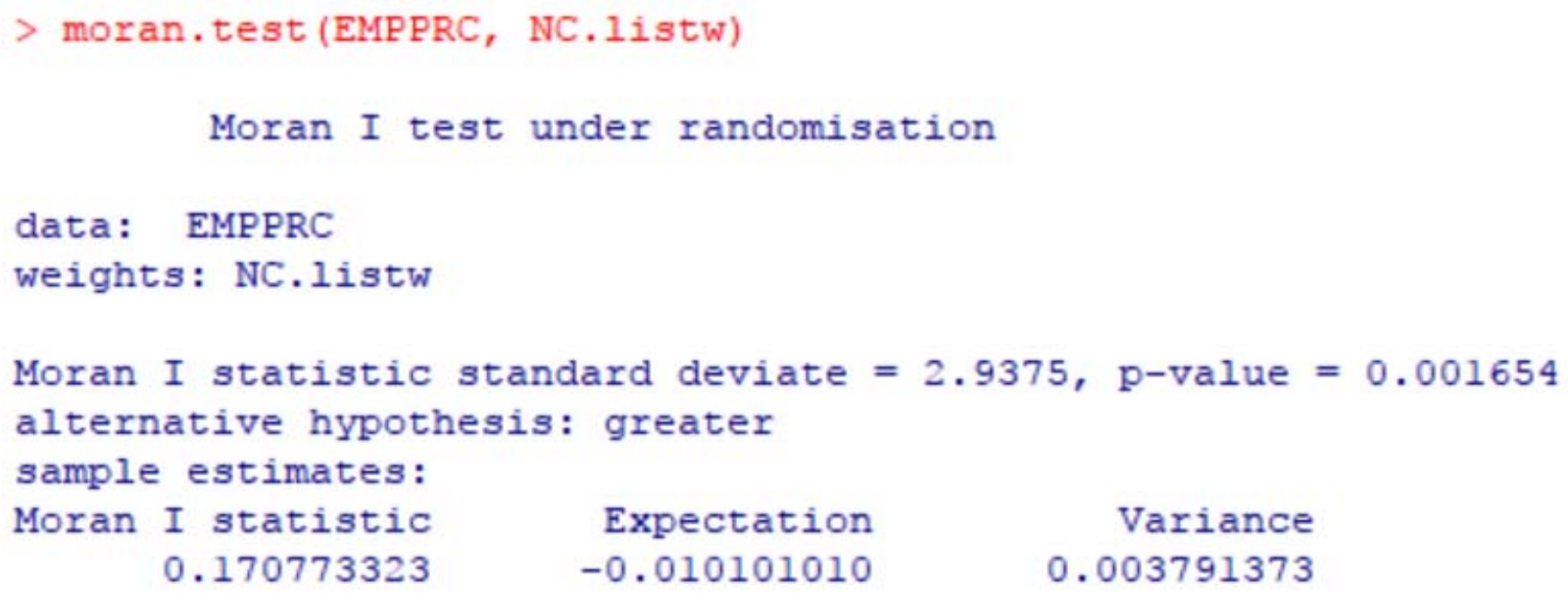

I also conduct a Geary's C test to make sure about the result which confirms the result from the Moran's I test.

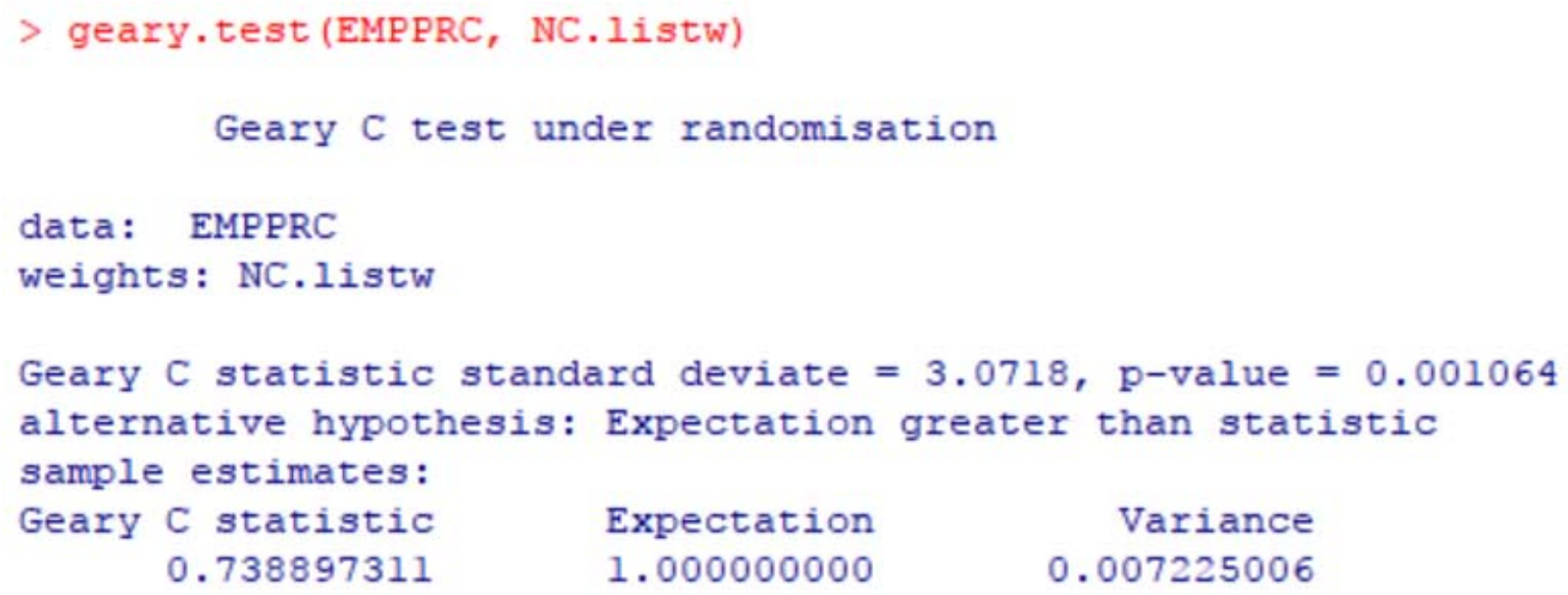

\section{Moran Scatterplot}

Scatterplot of the mean value of neighbors of percentage change in employment between 2010 and 2015 (EMPPRC) against the EMPPRC (Figure 1).

There are more High-High: high values surrounded by high values 
Low-Low: low values surrounded by low values in the scatterplot.

It means that those counties with higher Percentage Change in employment (EMPPRC) surrounded by counties that have high EMPRC and, those counties with low EMPPRC surrounded by neighbors with low EMPPRC.

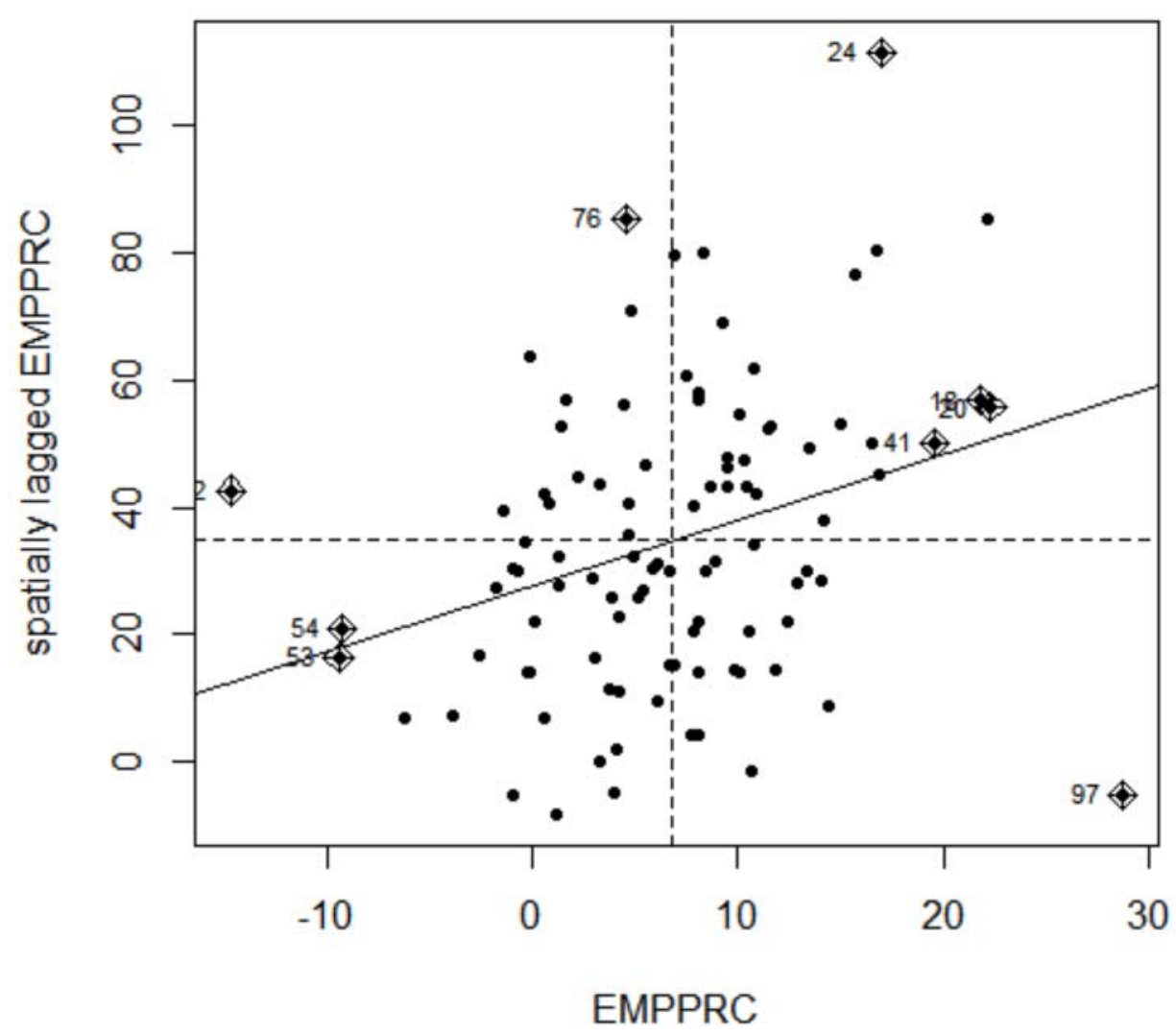

Figure 1 Moran Scatterplot

\section{Regression Analysis:}

Since we saw that we have spatial autocorrelation, now we can conduct a OLS analysis to see if there is any spatial dependency among the counties. In the following pages, you can see these analyses for one OLS that has EMPPRC as the dependent variable and two explanatory variables of per capita income in 2010 (INC2010_1) and share of manufacturing in 2010 (MANF2010_1). 
The results show that MANF2010_1 is significant in 10\% level, and INC2010_1 is significant in $5 \%$ level.

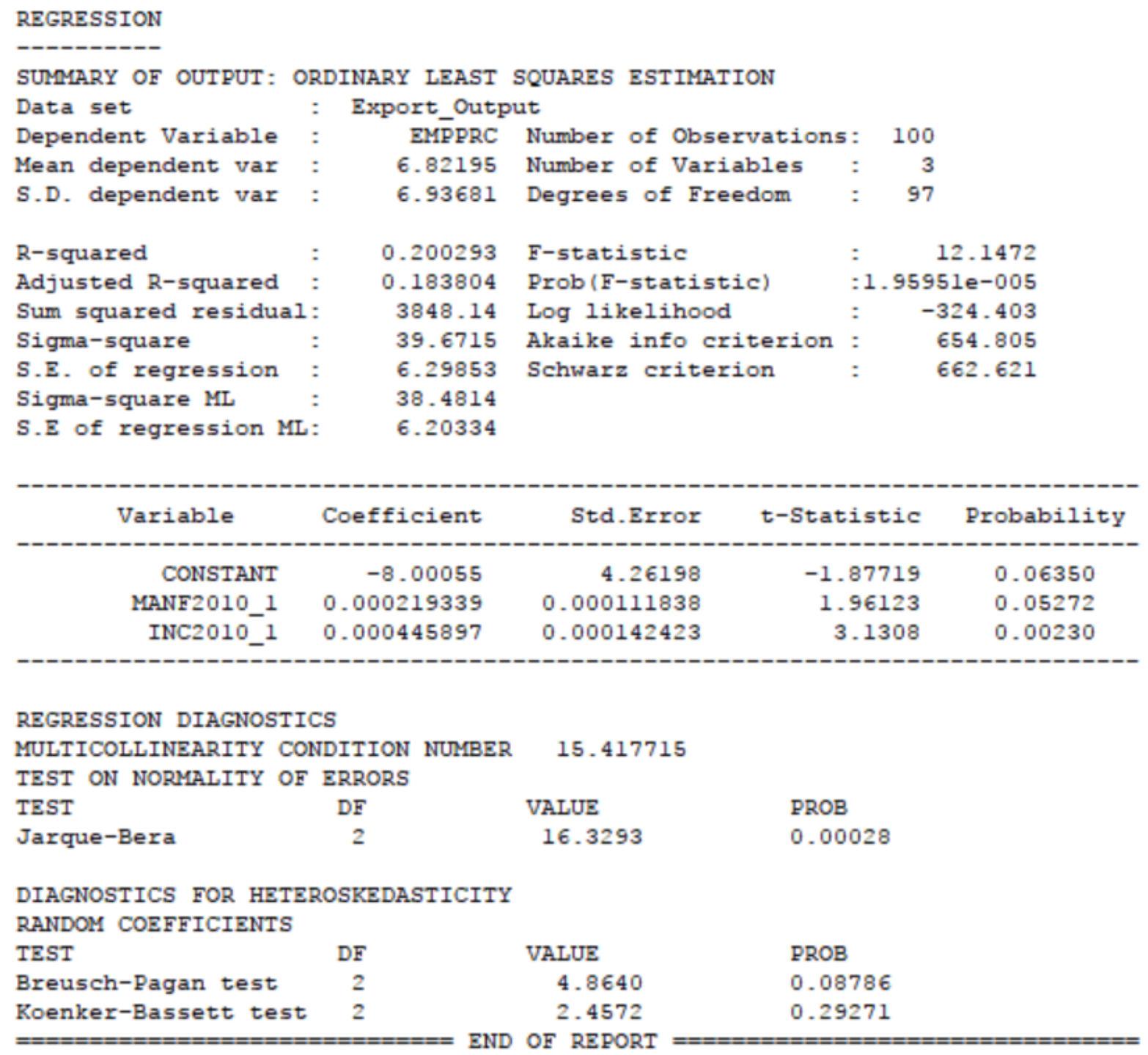

Then I calculated the residuals and ran a Moran's I test to see if there is any spatial dependency. However, since the line is almost perfectly horizontal, there is no spatial dependency and we do not need to do spatial lag model. And we can use the OLS (Figure 2). 


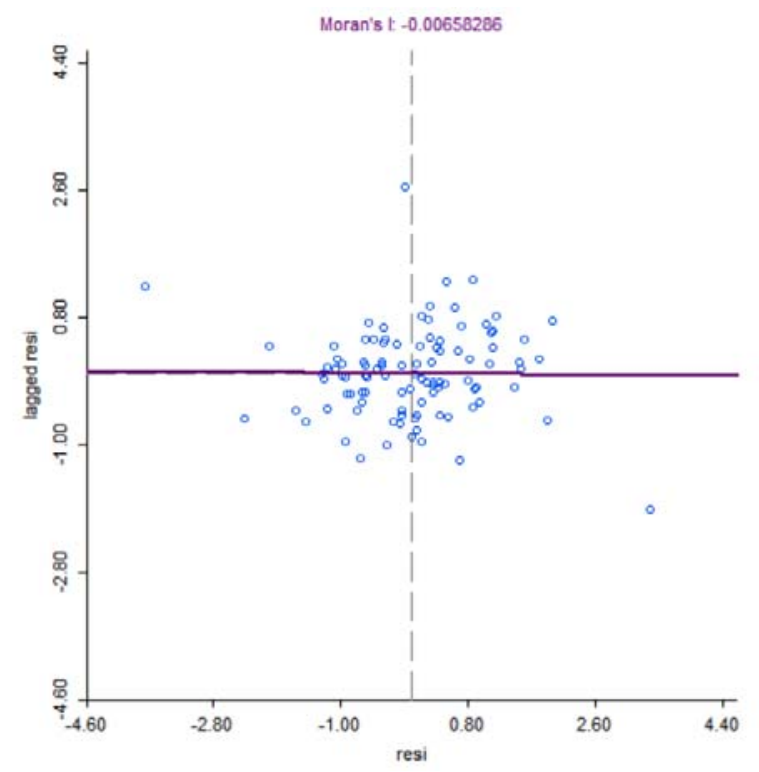

Figure 2 Moran's I test on Residuals of the OLS

\section{Local Moran's I on Percent Change in Employment between 2010 and 2015 (EMPPRC)}

Local Moran's I is used here to reveal the spatial structure of clustered data. We see in map 4 that there is clustering in NC counties based on the percent change in employment between 2010 and 2015.
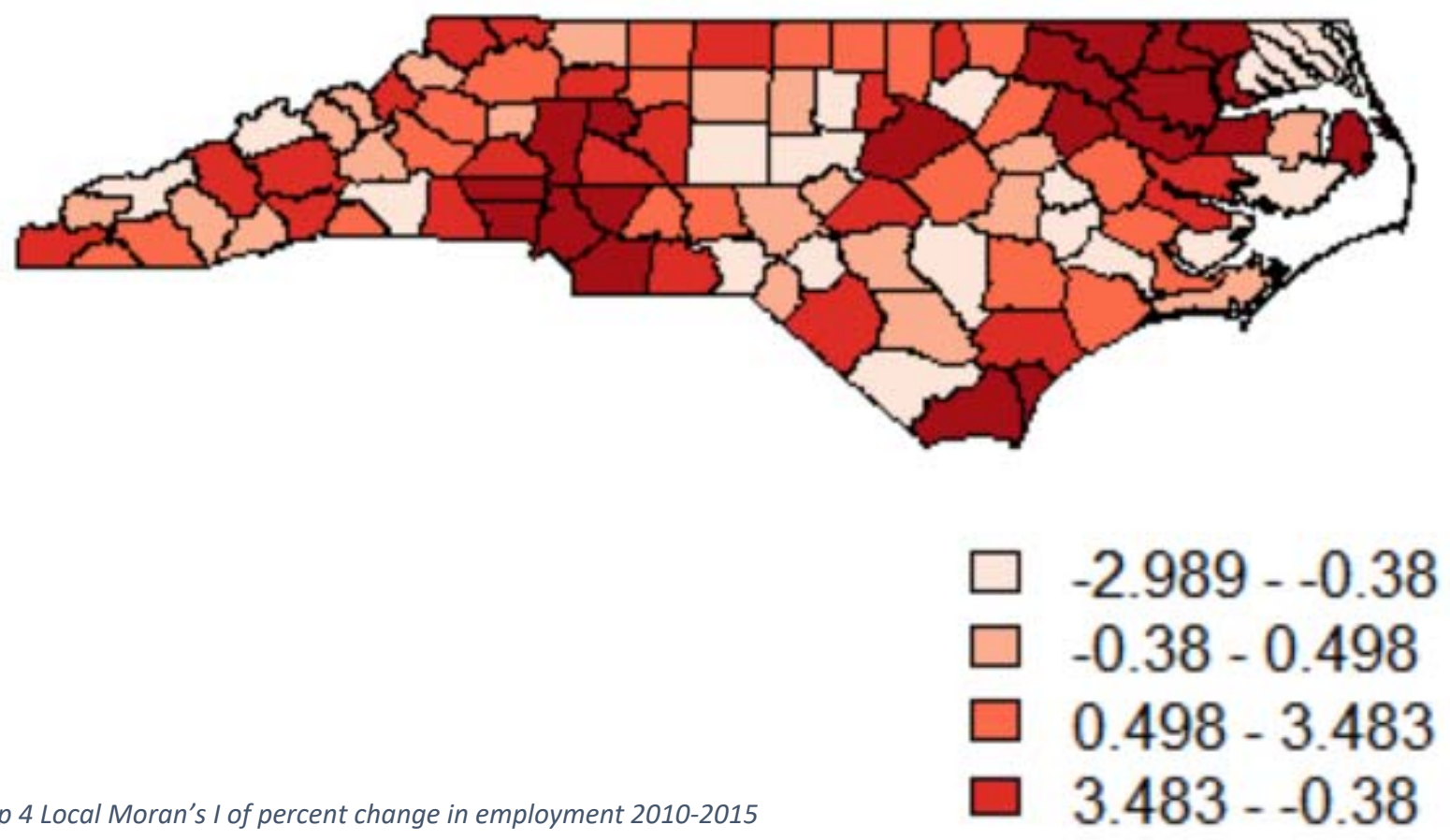


\section{Local Getis-Ord's G analysis}

In this section I used Getis-Ord's G method. The hotspots in Getis-Ord G shows areas that are related rather than whether they are surrounded by features with similar values in local Moran's I. The results of local Getis-Ord's G are Z score that tell where EMPPRC with either high or low values cluster spatially. It works by looking at each feature within the context of neighboring features. Those areas in the maps with darkest red are hot spots that the clusters are more intense. Also, those areas with brightest color are cold spots that the clusters have the least intensity.

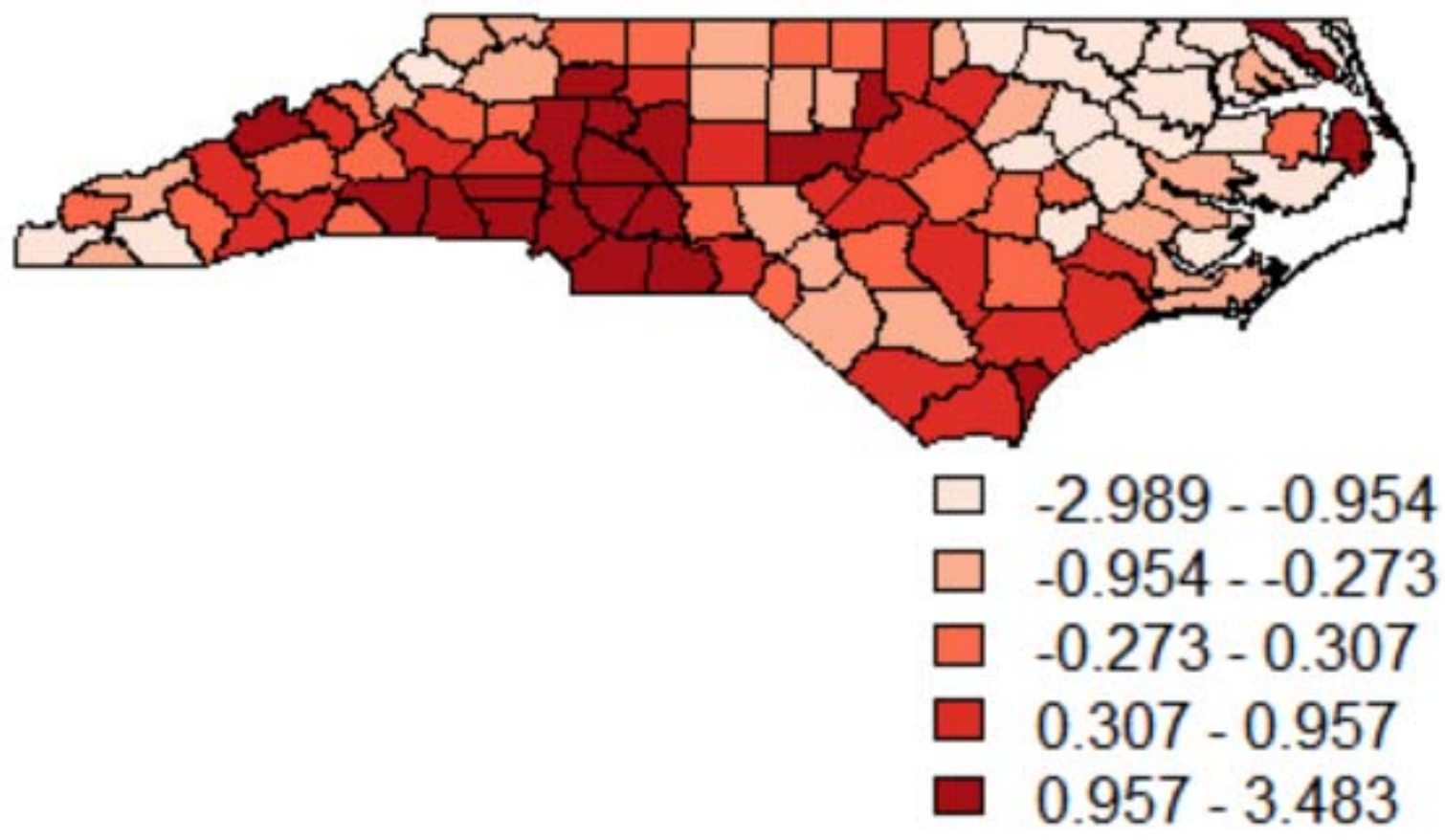

Map 5 Local Moran's I of employment in 2015 


\section{H. Conclusion:}

To sum up this study, I emphasize on the implication of this study for economic development policies. Based on this analysis, the adjacent counties have spatial autocorrelation with each other. Since, development is cross boundaries, we should be careful about the results of any changes in any area or region. When NC government decides about the allocations of funding for employment growth they can consider these spatial autocorrelations to make better decisions.

\section{References}

1. Anselin, L., and Da Griffith. "Do Spatial Effects Really Matter in Regression-Analysis." Papers of the Regional Science Association 65 (1988): 11-34.

2. Anselin, Luc. "Thirty Years of Spatial Econometrics." Papers in Regional Science 89, no. 1 (March 1, 2010): 3-25. doi:10.1111/j.1435-5957.2010.00279.x.

3. Cliff, Andrew, and Keith Ord. "Testing for Spatial Autocorrelation Among Regression Residuals." Geographical Analysis 4, no. 3 (July 1, 1972): 267-84. doi:10.1111/j.15384632.1972.tb00475.x.

4. Dubin, Robin A. "Spatial Autocorrelation: A Primer." Journal of Housing Economics 7, no. 4 (December 1, 1998): 304-27. doi:10.1006/jhec.1998.0236.

5. Elhorst, J. P., 2003. Specification and Estimation of Spatial Panel Data Models. International Regional Science Review, 26:244-268.

6. Getis, Arthur. "A History of the Concept of Spatial Autocorrelation: A Geographer's Perspective." Geographical Analysis 40, no. 3 (July 1, 2008): 297-309. doi:10.1111/j.15384632.2008.00727.x. 
7. Goetzke, Frank. "Network Effects in Public Transit Use: Evidence from a Spatially Autoregressive Mode Choice Model for New York." Urban Studies 45, no. 2 (February 1, 2008): 407-17. doi:10.1177/0042098007085970.

8. Goodchild, M., R. Haining, S. Wise, G. Arbia, L. Anselin, E. Bossard, C. Brunsdon, et al. "Integrating Gis and Spatial Data-Analysis - Problems and Possibilities." International Journal of Geographical Information Systems 6, no. 5 (September-October 1992): 407-23. doi: $\underline{10.1080 / 02693799208901923}$.

9. Haining, Rp. "Moving Average Model for Spatial Interaction." Transactions of the Institute of British Geographers 3, no. 2 (1978): 202-25. doi:10.2307/622202.

10. Jong, P. de, C. Sprenger, and F. van Veen. "On Extreme Values of Moran's I and Geary's c." Geographical Analysis 16, no. 1 (January 1, 1984): 17-24. doi:10.1111/j.15384632.1984.tb00797.x.

11. Khabazi, M. "Regional Economic Analysis of Mecklenburg County, NC" SocArXiv, 2018. doi: 10.17605/OSF.IO/H9ER2

12. Liu, Yu, Daoqin Tong, and Xi Liu. "Measuring Spatial Autocorrelation of Vectors." Geographical Analysis 47, no. 3 (July 1, 2015): 300-319. doi:10.1111/gean.12069.

13. Peeters, Aviva, Manuela Zude, Jana Käthner, Mustafa Ünlü, Riza Kanber, Amots Hetzroni, Robin Gebbers, and Alon Ben-Gal. "Getis-Ord's Hot- and Cold-Spot Statistics as a Basis for Multivariate Spatial Clustering of Orchard Tree Data." Computers and Electronics in Agriculture 111 (February 2015): 140-50. doi:10.1016/j.compag.2014.12.011.

14. Tobler, W. R. "A Computer Movie Simulating Urban Growth in the Detroit Region." Economic Geography 46 (1970): 234-40. doi:10.2307/143141. 
15. Wang, Chih-Hao, Gulsah Akar, and Jean-Michel Guldmann. "Do Your Neighbors Affect Your Bicycling Choice? A Spatial Probit Model for Bicycling to The Ohio State University.” Journal of Transport Geography 42 (January 2015): 122-30. doi:10.1016/j.jtrangeo.2014.12.003. 